\title{
Philo-sophía, bio-logos y bio-techne
}

\section{María Teresa Padilla Longoria}

Juliana González V., coord., Filosofía y ciencias de la vida. México, UNAM, Facultad de Filosofía y Letras/FCE, 2009. 276 pp.

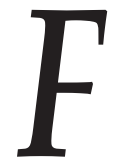

ilosofía y ciencias de la vida, coordinado por Juliana González, es un libro que reúne catorce artículos relativos al entretejimiento -hoy por hoy insoslayable- de problemas y temáticas de la filosofía, las ciencias de la vida y la biotecnología. Los catorce textos reelaborados y ahora coeditados por nuestra casa de estudios y el Fondo de Cultura Económica, fueron originalmente presentados y discutidos en los "Entretiens" del Instituto Internacional de Filosofía, celebrados en Cuernavaca en el mes de agosto de 2005. Once de ellos son de los miembros de dicho Instituto y los tres restantes de filósofos y científicos mexicanos invitados a dicho acto.

La obra está dividida en cuatro bloques temáticos:

El primero enfrenta, sin ambages, "Los grandes temas", a saber: vida, conciencia y razón. Abre con el trabajo de Pierre Aubenque, “iLo vivo como modelo? Acerca de los límites de la interpretación biológica en el pensamiento clásico griego". El filósofo francés presenta un escrito sugerente y estimulante. Su hipótesis de trabajo es que: "Lo vivo es uno de los modelos que el pensamiento clásico griego utilizó para reflexionar en su conjunto sobre la génesis y la organización del universo" (p. 19). Al no ser el único paradigma, permite - a juicio de Aubenque- ponderar sus alances y límites. Como buen estudioso de Aristóteles, Aubenque nos presenta, principalmente, las críticas que el estagirita hiciera a los arquetipos explicativos presocráticos y las insuficiencias de las que éstos adolecen, a saber, mecanicismo e hilozoísmo. Nos hace notar las ventajas del modelo biológico de Aristóteles -con antecedentes y expresiones más que claras en su maestro Platón-: 1. La proximidad con algo vivo como nosotros y sometido a los mismos procesos de nacimiento y muerte, y 2. La correlación y prioridad teleológica de la biología, es decir, que epistemológicamente -e incluso estéticamente- explica los fenómenos recurriendo a las causas finales involucradas. 
El gran mérito de la biología aristotélica es -según nos argumenta Aubenque- el unir esencia y finalidad, es decir, la causa formal y la final de manera interna en su concepción del ser vivo. No obstante, para las ciencias moderna y contemporánea, la teleología está a la baja como forma explicativa de los fenómenos y del conocimiento. Pero el filósofo francés insiste en que el biologismo de Aristóteles culmina en su definición del alma como principio

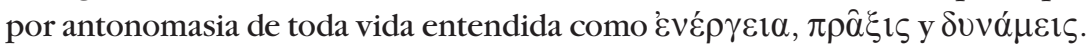
Pero, se pregunta el propio Aubenque, si esta definición se restringiera al alma humana en exclusiva y no se hiciera extensiva a los demás seres vivos e incluso análogamente a los demás seres físicos, ¿qué valor tendría? Por esto mismo el estagirita nos proporciona una definición más general de la vida referida a los seres naturales como aquellos que contienen en sí mismos el principio de movimiento y de reposo. ${ }^{1}$

Aubenque ve en estas generalizaciones y oscuridades definitorias en Aristóteles acerca de lo que debe entenderse por alma y por vida, faltas de rigor en su biología. De hecho, nos esclarece, que ésa es la razón por la que el propio estagirita objetará la extrapolación del modelo biológico (animista) a la cosmología -que parece, a juicio del propio Aristóteles, ser la tesis sostenida por Platón- por haber incompatibilidad entre el supuesto de un alma divina y la eternidad del movimiento celeste. En consecuencia, en relación con la explicación del movimiento del Primer cielo, Aristóteles dará prioridad al modelo explicativo físico-matemático por corresponder mejor al carácter trascendente del elemento astral y por no introducir en el Primer principio características que le son impropias y que nos son más que indicaciones de antropomorfismo. Llama nuestra atención que Aubenque mismo nos dice que Aristóteles seguirá hablando de un Dios como un ser vivo, pero con una vida no biológica, suprasensible y como acto puro.

En conclusión: en conformidad con la hermenéutica de Aubenque, no es posible hablar de un paradigma biológico dominante en Aristóteles que pueda emplearse como explicación universal. Considera que tanto en el estagirita como en su maestro Platón el modelo dominante, aunque no exclusivo, fue el matemático. No por ello, debe dejarse de reconocer-hace hincapié el filósofo francés- que la aplicación del modelo biológico de Aristóteles al mundo de lo moviente constituye un antecedente remoto del paradigma epistemológico de la biología moderna.

A nuestro juicio, y con todo respeto para el profesor Aubenque, la idea griega de la $\varphi v ́ \sigma ı \varsigma$, con la totalidad del sentido de dinamismo y proceso que entraña una génesis, el proceso mismo y los resultados, nos permite concluir que es justamente el modelo físico-matemático el que debe ser subsumido

\footnotetext{
${ }^{1}$ Vid. Aristóteles, Física, II, 192b 13.
} 
por el biológico y que hay fundamentos para hablar de una bio-physis universal en el pensamiento helénico que sería la que los contemporáneos deberíamos recuperar y restituir como base para las investigaciones bioéticas y biotecnológicas.

El segundo análisis es el de Jesús Mosterín titulado “¿Qué es la vida”. El autor parte de la tesis de que la vida es un hecho milagroso. Iniciando con la noción aristotélica de la vida -que a juicio del filósofo español fue el único pensador de la Antigüedad clásica en desarrollar una filosofía de la vida- despliega toda una explicación de la bioquímica de la misma pasando por las ideas de la individualidad paradigmática y elemental de la célula, la complejidad genómica en el problema de la definición de la vida, el carácter teórico-histórico de toda ciencia, las bases alternativas para la vida, el carbono y el silicio como unidades estructurales, el agua y el amoniaco como solventes, los aminoácidos como componentes estructurales de las proteínas, el trifosfato de adenosina como la clave por antonomasia de la vida y la célula eucariota como el eslabón más complejo en la evolución de la vida terrestre. Mosterín concluye con la siguiente afirmación: "La sabiduría requiere de autoconciencia. Para saber quiénes somos, qué clase de lugar es el universo y en qué medida somos milagros únicos con ciclos regulares, necesitamos responder la pregunta acerca de la existencia y el carácter de la vida en otros lugares" (p. 59).

El tercer estudio es el de John R. Searle titulado "La conciencia". El trabajo es inteligente y proponente. Al comienzo, nos indica cómo el interés por el estudio de la conciencia data, tan sólo, de veinte años atrás. En particular se centrará en la caracterización filosófica de algunos de los problemas neurobiológicos de la conciencia. El problema fundamental se plantea en términos de la correlación entre los procesos cerebrales y la generación de estados conscientes, o más específicamente, en qué consisten los correlatos neurobiológicos de la conciencia y a qué nivel deben explicarse.

Searle pone énfasis, desde un principio, en que la conciencia debe ser investigada y entendida como un problema y, por ende, como fenómeno biológico con un trasfondo constante de problemas filosóficos. Dicho trasfondo filosófico entraña el rigor de definir a la conciencia como una diversidad de estados y de procesos de índole interna, subjetiva, que involucran a la sensación y por lo cual nos percatamos de algo.

A juicio de Searle, la mejor forma de explicar la conciencia es la neurobiológica, pues ésta nos permite mostrar su característica esencial, a saber, la implicación mutua y combinación de su naturaleza cualitativa, subjetiva -ontología de primera persona- y unitaria. O en palabras del propio Searle: “...El problema es explicar cómo es que los procesos cerebrales, que son procesos biológicos, químicos y eléctricos objetivos de tercera persona, producen estados subjetivos de sensación y pensamiento" (p. 69). 
Menciona otras características de la conciencia: la intencionalidad de los estados conscientes, la distinción entre el centro y la periferia de la atención, la integración de todas las experiencia humanas conscientes a algún estado de ánimo u otro, a una dimensión placer/displacer, y a una estructura perceptiva coherente de los estímulos (estructura Gestalt) y la familiaridad en las experiencias conscientes.

Para evitar el problema mente-cuerpo propone un naturalismo biológico que explica la conciencia ontológicamente subjetiva y supera los dualismos y materialismos a este respecto.

Concluye con una tesis holística, es decir, que hay un único campo consciente unificado del cual se derivan los diferentes aspectos sensoriales. Censura fuertemente las aproximaciones al estudio de la conciencia que aseveran que ésta no es más que un tipo especial de programa computacional o de procesamiento de información. En contraposición, Searle propone que, con vistas a la comprensión de la naturaleza de la conciencia, es necesario entender el cómo de los procesos cerebrales que originan y realizan la conciencia misma.

A nuestro juicio, el profesor Searle se vería profundamente sorprendido por las aportaciones y luz que a este respecto le podrían dar el retorno a los griegos, en particular, el Sofista y Filebo de Platón, el De anima y De la memoria y el recuerdo de Aristóteles y la Carta a Heródoto de Epicuro, entre otras obras de la Antigüedad clásica.

El primer bloque cierra con el trabajo de Bertrand Saint-Sernin titulado "La racionalidad científica a principios del siglo XXI". Con todo respeto para el profesor Saint-Sernin, el título no corresponde a lo que desarrolla. Más bien nos expone qué condiciones externas deben darse para que el trabajo interdisciplinario pueda ser teóricamente consistente y fiable y cómo la producción del saber científico requiere - hoy por hoy- la participación de agentes colectivos. A su juicio, se está constituyendo una nueva racionalidad basada en las relaciones intersubjetivas en donde ciencia, moral y política están entrelazadas profundamente. Dicho racionalismo, por ser de índole colectiva, requiere instituciones para su realización.

El segundo bloque lleva por título "De la filosofía a las biociencias, de las biociencias a la filosofía” y contiene tres análisis:

El primero, de Anne Fagot-Largeault, referente a las "Preguntas que las ciencias de la vida hacen a la filosofía" nos hace ver que el diálogo entre filósofos y científicos no ha sido fácil en lo absoluto. Sin embargo, desde la segunda mitad del siglo $\mathrm{xx}$, las ciencias de la vida han originado una serie de interrogantes a los filósofos, las cuales les han exigido que profundicen y aprendan de este ámbito científico. Entre las preguntas de fondo se hallan las siguientes: ¿cuáles son los límites de la utilización del mundo vegetal y animal como objetos de investigación con vistas a las necesidades humanas? ¿Cuál es la misión y la responsabilidad 
de la especie humana ante la naturaleza y el proceso evolutivo universal al que pertenece? ¿Se deben poner límites morales a la experimentación?

Fagot-Largeault propone que la filosofía de la ciencia no debe ser concebida como una lógica de la ciencia, sino como una filosofía de la naturaleza. A su juicio, esta nueva filosofía de la ciencia ha recibido un renovado impulso gracias a las ciencias de la vida y al trabajo conjunto -y mutuo afán de aprendizaje y discusión- entre los biólogos y los filósofos.

Finalmente deja planteada la pregunta abierta de si será posible que se constituya una comunidad internacional de investigadores de filosofía de las ciencias de la vida, como ya se da la de investigadores en ciencias de la vida.

El segundo artículo es de Juliana González y se refiere a la "Ontología y ciencias de la vida". La filósofa mexicana nos hace ver que la época actual constituye una oportunidad excepcional para el reencuentro entre las ciencias y la filosofía y, particularmente, entre esta última y las ciencias de la vida.

Este reencuentro es cuestión de corresponsabilidades, ya que las biociencias no pueden hacer caso omiso de las consecuencias éticas, axiológicas y sociohistóricas de sus pesquisas y alcances tecnológicos, ni la filosofía puede evadir las transformaciones científicas, tecnológicas y ontológicas de tales derivaciones.

J. González indica, atinadamente, que la bioética es el lugar de convergencia multidisciplinaria por antonomasia entre bios, ethos y ethiká. Plantea la gran paradoja que comporta la pregunta por el cómo tener acceso al sustrato ontológico derivado del problema por la púoıৎ metafísica del ser humano si nuestros tiempos son posmetafísicos.

Es aquí cuando se hace necesario asumir en otros términos no reduccionistas -llámense esencialismo o nihilismo- los hallazgos de las biociencias y comprender la naturaleza de la vida desde su hondura, complejidad y raigambre biológica hasta sus connotaciones metafísicas relativas también a cuestiones fundamentales como la unidad y la diversidad de la materia viva, el porqué de su permanencia y de su cambio, de su ser y devenir y de su impronta a la vez necesaria y contingente.

J. González insiste en el carácter salvador de la biología con respecto a la filosofía, ya que la revolución genética que aquélla generó - por la revelación de la molécula del ADN- hizo manifiesto "el sustrato universal de la vida" como principio de unidad y diversidad de todo lo vivo. Pero, por esto mismo, pone énfasis en el carácter plástico, complejo y dinámico del genoma humano que nos lleva a la conclusión de que lo epigenético también cuenta y cuenta mucho. O en palabras de la propia filósofa:

[...] La vida conlleva el misterio de cómo la materia se hace vida sin dejar de ser materia y sin reducirse a pura materia, es decir, el misterio de 
ese "salto" que se da sin ruptura, sin quiebra de la esencial continuidad: el radical cambio cualitativo (ontológico) de la materia a la vida que no rompe con su base material y el misterio de cómo la vida se hace cultura, historia, "espíritu", sin dejar de ser vida natural (p. 129).

Es por eso que J. González apela a la teoría de la emergencia como puente entre la biología y la ontología, a saber, que todo proceso genético es siempre palingenésico. Es a partir de esta nueva premisa que se podría construir un

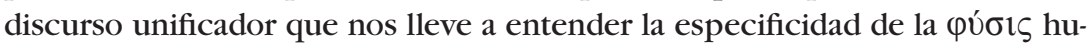
mana inmersa en la biophysis universal.

La tercera exploración - con la que concluye la segunda parte- corresponde a Evandro Agazzi y se titula "Una nueva oportunidad de diálogo entre ciencia y filosofía". El filósofo italiano nos muestra cómo, sin duda alguna, la ciencia natural moderna tuvo un impacto innegable sobre la filosofía, pero el ideal de la ciencia físico-matemática como modelo único y definitivo a seguir ha caído por tierra. Esto también se ha derivado de la noción de emergencia proveniente de las ciencias de la vida que nos ha mostrado un tipo de realidad ontológicamente diferente y no reducible -si es posible expresarlo así- a la pura materia. Esto ha reabierto el panorama, orientación y vinculación de la filosofía de las ciencias de la vida con y hacia la ontología, la filosofía de la naturaleza y la antropología filosófica y originado, así, un nuevo diálogo entre filosofía y ciencias. Agazzi concluye que el desarrollo de las ciencias de la vida ha llevado también a acabar con la antigua idea de su neutralidad cognitiva y a abrir un diálogo insoslayable sobre cuestiones inéditas con la ética para a hablar, entonces, de un renacimiento de la ética en nuestro siglo.

La tercera parte de la obra trata acerca de las Cuestiones de bioética y tecnociencias y contiene cinco investigaciones:

La primera de Hans Lenk, "Albert Schweitzer como un pionero de la bioética”: tal y como el título nos lo indica, Lenk señala que, Schweitzer, a partir de su famoso lema y principio básico "Ehrfurcht vor dem Leben" (reverencia, profundo respeto por la vida), sienta las bases racionales de la bioética recurriendo a un proceso deductivo para establecer valores y normas éticas. Con un tinte un tanto schopenhaueriano, Schweitzer pone énfasis en la individualización de la voluntad de vivir como experiencia primordial que se hace extensiva y, por ende, adquiere carácter universal para todos los otros seres vivos.

Como bien destaca Lenk: "...la fundamentación de la ética de Schweitzer, es bioética per se en el sentido amplio de la palabra" (p.153). Asimismo, el académico alemán, subraya que Schweitzer, no sólo sistematiza el respeto a todos los seres vivos, sino que también pone en evidencia varios de los problemas, principios prácticos y decisiones relativas a la "ruptura en la naturaleza". 
Las soluciones que Schweitzer propone a este respecto, vía la materialización práctica de sus principios, apelan a la conciencia personal bien forma$\mathrm{da}$, atenta y crítica con base en los derechos morales de todo ser vivo. O tal y como lo vierte la propia interpretación de Lenk al respecto:

“¡Nadie debe tomar su responsabilidad a la ligera!” La ética no puede ser una lista completa de instrucciones casuísticas para actuar en cualquier situación, tampoco un mecanismo deductivo para derivar consecuencias explícitas de una decisión y acción a partir de los más altos principios éticos, tampoco hay que tomar la responsabilidad y el cultivo de la propia conciencia a la ligera. Por el contrario, de acuerdo con Schweitzer, la ética es una empresa seria para aplicar prácticamente y desarrollar nuestra propia libertad moral, dignidad humana, conciencia ética y empatía con todas las formas de vida, incluyendo las no humanas. Podemos ver nuestra libertad moral y nuestra autonomía en esta responsabilidad personal e individual, en esta siempre vigilante reflexión bajo la máxima de la reverencia por la vida (p. 156).

La segunda pesquisa, de Peter Kemp, "La reciprocidad entre bioética y bioderecho", destaca la acuñación reciente (década de los noventas) de este último como vocablo - pero en estrecha vinculación y derivación del primero. Por bioderecho se entiende el marco de referencia irrenunciable, tanto legal como administrativo, ante el surgimiento de las nuevas biotecnologías con vistas a la protección de los seres humanos. De esta forma, el bioderecho es una expresión de la filosofía del derecho cuyo propósito consiste en el examen de las cuestiones legales insertas en materia de bioética y biotecnología.

Kemp hace hincapié en que una de las tareas fundamentales de la bioética es proporcionar los cimientos y razones para la protección del cuerpo humano frente a la manipulación biomédica o biotecnológica. Asimismo, es primordial clarificar lo referente al comienzo y fin de la vida humana, no sólo para enfrentar las cuestiones de la eutanasia y del estatus jurídico del embrión humano, sino también para dotar de principios y razones éticas y de marcos jurídicos a la intervención tecnológica y química en el cuerpo de los seres vivos. Sucintamente: ética y derecho, y particularmente, bioética y bioderecho deben trabajar de manera coordinada para el conocimiento, respeto y protección de la naturaleza en su conjunto, con miras a los antiguos, pero no por ello menos actuales, idea e ideal platónicos de la justicia y la del bien vivir.

El tercer trabajo de Gilbert Hottois se titula: “QQué papel deben desempeñar los filósofos en los debates bioéticos? Presentación y discusión de $E l$ futuro de la naturaleza humana. ¿Hacia una eugenesia liberal?, de Jürgen Habermas. 
Hottois expone, con detalle, la posición y argumentos de Habermas, a los que, sin dejar de respetar, terminará calificando como un tanto conservadores. Inicia destacando el peligro que el filósofo alemán ve en las intervenciones genéticas, específicamente, en la posibilidad de una eugenesia liberal o privada, controlada por el mercado, que genere una manipulación no lingüística sobre las condiciones mismas de posibilidad de los individuos para tener acceso al estado particularmente humano de "sujetos comunicativos", con la consecuente capacidad de deliberación y elección individual e intersubjetiva. Ante este riesgo, Habermas propone una defensa de posiciones sustancialistas y un derecho que propugne por una normatividad protectora. La tesis fuerte de Habermas es - a juicio de Hottois- la reafirmación de los vínculos entre moral, derecho y política vía la legalidad jurídico-política de la ética, mejor conocida como su "Gattungsethik", ética de la especie humana.

Habermas ha definido su postura como una reflexión crítica sobre la modernidad con base en las propias condiciones que la hacen posible y cuya defensa es central para ir en contra de los espejismos y falacias que sustentan la manipulación de la naturaleza interna - el cuerpo, el cerebro y el genoma humanos- en aras de una libertad tecnocientífica.

En la segunda sección de su obra, Habermas discute en torno al estatus del embrión humano y defiende una postura continuista y gradualista que reconoce tanto en el embrión como en el feto un valor intrínseco y creciente que va más allá de éticas individualistas o comunitarias.

En la tercera sección de la obra del filósofo alemán, "La inserción de la moral en la ética de la especie" - nos comenta Hottois- reafirma la tesis de que la mayoría de los planteamientos habermasianos son de orden lingüístico, simbólico y hermenéutico-discursivo y que el ser humano tiene el derecho inalienable de seguir siendo un ser vivo simbólico.

Las restantes cuatro secciones de la obra hacen hincapié en lo anterior. Habermas acepta la eugenesia negativa, a saber, la realizada con vistas al "reforzamiento del sistema inmunológico o la prolongación de la vida" (p. 187). O como lo expresa el propio Hottois: "Según Habermas, el hecho de saberse genéticamente programado (incluso aunque no haya determinismo genético) constituye un obstáculo simbólico al advenimiento consciente de la autonomía personal" (p. 188).

En la parte final, Hottois lleva a cabo una discusión crítica, tanto de las tesis, como de los argumentos de Habermas. Indica que tiene un doble desacuerdo con el filósofo alemán: en los contenidos de su filosofía y en el papel que él le asigna a ésta. Piensa, además, que sus argumentos adolecen de ambigüedad, pues recurren a una petición de principio basada en una concepción determinista y vaga de lo que él entiende por genética. Asimismo, ve en los planteamientos habermasianos razonamientos radicales expresados en tér- 
minos de todo o nada. A su juicio, es preferible optar por una regulación de la libre investigación, y permitir bajo vigilancia procedimental rigurosa, pública y revisable los experimentos que muchos otros, simplemente, prohíben de manera tajante. Hottois también considera que las ejemplificaciones e intuiciones de Habermas son parciales, pues descalifica -o incluso ignorade manera sistemática los usos eugenésicos positivos. Aún más: Habermas presenta contraposiciones conceptuales cuestionables, particularmente, entre el condicionamiento genético y el simbólico. Esto lo lleva a apelar a recursos afectivos, emocionales y sentimentales que pueden generar intuiciones y prejuicios discriminatorios sumamente arriesgados.

Hottois también considera que Habermas hace uso recurrente de argumentos de índole psicológica y sociológica y predispone, de esta manera, a generar obstáculos a prácticas -ya legítimas- por considerarlas contrarias a la moral. De ahí que Hottois concluya que el pensamiento habermasiano no sea más que una prolongación de la larga tradición de la filosofía idealista ligada a la metafísica y a la religión. En suma: el discurso habermasiano, aunque muy respetable, es teórico, apriorista y cerrado al diálogo.

La cuarta intervención, "Ciencias, tecnociencias y humanidades", corresponde a Ambrosio Velasco, quien sostiene la tesis de que es necesario conformar un nuevo modelo epistemológico científico-humanístico y tecnológico que integre episteme, phrónesis y techné. Resalta, entonces, que la revolución de las ciencias genómicas tiene consecuencias filosóficas y establece una unión natural, a manera de continuidad ontológica, entre materia, vida y cultura.

El tercer bloque cierra con el trabajo de Evandro Agazzi, "La tecno-ciencia y la identidad del hombre contemporáneo", que es una crítica dirigida a las interpretaciones mecanicistas del ser humano. El filósofo italiano basa su examen en la insuficiencia de la máquina como modelo de inteligibilidad y prototipo de las ciencias naturales modernas que eliminaron la categoría de finalidad.

Esto desencadenó una serie de consecuencias nada desdeñables. Ahora se juzga que los modelos artificiales son de tal eficacia que ya tienen un estatus ontológico que no se puede diferenciar de los sistemas naturales y que por eso nos proporcionan los principios ontológicos explicativos de estos últimos. Por eso Agazzi pone énfasis al respecto:

Si se aplica esta estrategia a la creación de modelos para los sistemas naturales -como los hombres-capaces de realizar actividades llamadas mentales que van de la percepción a la memoria, al razonamiento deductivo, a la abstracción y a la comunicación verbal, entonces se puede comenzar a construir máquinas que sean capaces de realizar concretamente las mismas funciones que los seres humanos y, por esta vía se puede llegar a afirmar que dichas funciones no requieren la existencia 
de una sustancia inmaterial -como el espíritu- para comprenderlas y explicarlas. De esta manera, el espíritu se reduce a un epifenómeno de la materia y ésta, una vez que ha alcanzado el nivel de complejidad necesario gracias a las dinámicas del azar y la necesidad que ahora se han vuelto muy claras, se vuelve capaz de pensar (pp. 223-224).

Agazzi es contundente: estos modelos artificiales dejan de lado lo más propiamente humano, a saber, nuestra dimensión interior y, en el seno de ésta, la intencionalidad y la moral. Si todo en el ser humano se reduce a meros mecanismos internos o externos y nuestras acciones son simples comportamientos condicionados, entonces, ya no es posible hablar de mismidad humana, esto es, de conciencia de la propia libertad y de libertad de acción como vida proyectiva en la responsabilidad y creatividad.

Para escapar de los riesgos de la tecnociencia, el filósofo italiano propone dos vías complementarias entre sí: 1. La recuperación de la visión teleológica, proveniente de la tradición metafísica y teológica, que veía la doble finalidad de la naturaleza misma, tanto la inherente a todo ser individual, proveniente de su propia esencia, como la correspondiente a la confluencia de todas estas finalidades en la armonía de un orden natural universal, y 2 . La recuperación, primeramente, de la dimensión de la moralidad per se, que comporta una reivindicación de la interioridad objetiva y de proyección hacia la trascendencia $\mathrm{y}$, con base en esto, dirigirla al mundo tecno-científico.

Filosofía y ciencias de la vida concluye con la sección dedicada a la genómica y la biotecnología en México. Contiene los trabajos de dos científicos mexicanos, Gerardo Jiménez Sánchez y Francisco Bolívar Zapata. El primero de ellos, relativo a las "Oportunidades y retos de la era genómica", se centra en el tema del genoma humano y sus aplicaciones médicas, así como en sus variaciones en las poblaciones del mundo. Culmina con una exposición sobre el desarrollo y las perspectivas de la medicina genómica en México y los retos éticos, legales y sociales que ella implica. Destaca que el desarrollo de las ciencias genómicas y, específicamente, de la medicina genómica, constituye un nuevo arquetipo para la medicina moderna. Considera, por ello, previsible que la medicina genómica origine gradualmente aplicaciones que, a la larga, lleguen a constituir parte de la práctica médica habitual.

El segundo, referente a la "Biotecnología y bioseguridad para el desarrollo global y nacional" expone que debido a la biotecnología es posible reducir el tiempo requerido para verter los descubrimientos básicos en aplicaciones prácticas. A juicio de Bolívar Zapata, con una biotecnología mejorada y responsable se generarán oportunidades de producción ambientalmente solventes. Sin negar ni aminorar los riesgos y malos usos y abusos que entraña la construcción de organismos transgénicos, el científico reitera que si los propósitos de 
la misma son definidos, en tanto se hacen con vistas a mejorar nuestro conocimiento, a comprender mejor la naturaleza, a luchar contra las enfermedades y a enfrentar necesidades, los riesgos de descartar su uso son todavía mayores. Sobre todo porque nos condenarían a una dependencia tecnológica y a una pérdida de soberanía con los países en desarrollo. 